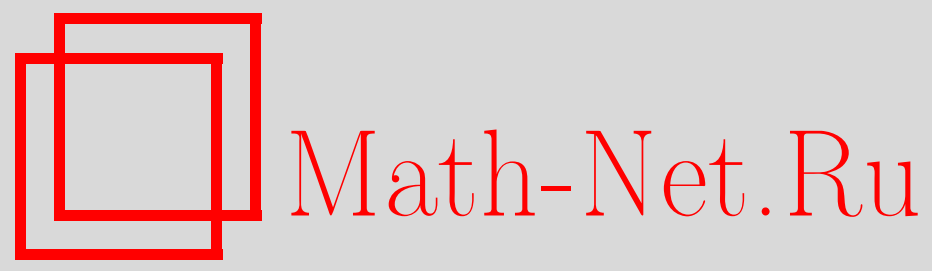

В. В. Манако, Представление температурного поля для полубесконечного тела, нагреваемого неподвижным лазерным лучом, через гипергеометрические функции, Вестн. Сам. гос. техн. ун-та. Сер. Физ.мат. науки, 2012, выпуск 2(), 115-123

DOI: https://doi.org/10.14498/vsgtu1017

Использование Общероссийского математического портала Math-Net.Ru подразумевает, что вы прочитали и согласны с пользовательским соглашением

http://www.mathnet.ru/rus/agreement

Параметры загрузки:

IP : 54.205 .225 .156

26 апреля 2023 г., 05:15:27

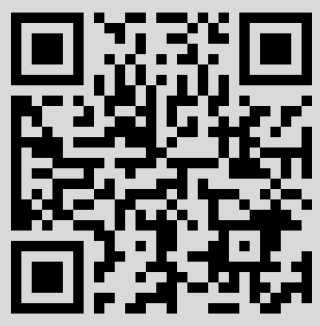


Вестн. Сам. гос. техн. ун-та. Сер. Физ.-мат. науки. 2012. № 2 (27). С. $115-123$

УДК 517.588:(517.958:536.212)

\title{
ПРЕДСТАВЛЕНИЕ ТЕМПЕРАТУРНОГО ПОЛЯ ДЛЯ ПОЛУБЕСКОНЕЧНОГО ТЕЛА, НАГРЕВАЕМОГО НЕПОДВИЖНЫМ ЛАЗЕРНЫМ ЛУЧОМ, ЧЕРЕЗ ГИПЕРГЕОМЕТРИЧЕСКИЕ ФУНКЦИИ
}

\author{
В. В. Манако \\ Самарский государственный технический университет, \\ 443100, Россия, Самара, ул. Молодогвардейская, 244. \\ E-mail: viktor.manako@mail.ru
}

\begin{abstract}
Рассматривается аналитическое выражение для нестационарного температурного поля в полубесконечном теле, нагреваемом круговым источником тепла, расположенным на свободной поверхности. Нестационарное поле температур выражается через гипергеометрические функиии Аппеля и Сриваставы. Рассматриваются частные случаи нестационарного температурного поля, которые выражсаются через функиию Кампе де Феръе. Полученные выражения позволяют сразу отделить стационарную часть температурного поля от нестационарной. Рассчитаны стационарные температурные поля, образуемые круговым и гауссовым источниками. Существенных количественных различий в этих полях не обнаружено.
\end{abstract}

Ключевые слова: краевая задачи теплопроводности, круговой источник тепла, гипергеометрические ряды.

Постановка задачи. Пусть на поверхность $z=0$ однородного и изотропного тела, занимающего полупространство $z \geqslant 0$, нормально падает поток лазерного излучения, создающий плоское пятно нагрева в виде неподвижного круга радиусом $R$. Через это круглое пятно рассматриваемое тело получает подводимую извне энергию лазерного излучения, а на остальной части поверхности $z=0$ теплообмен с окружающей средой отсутствует (адиабатическая граница). Воспользуемся цилиндрической системой координат $(r, \varphi, z)$, у которой начало отсчёта совпадает с центром пятна нагрева, ось $z$ совпадает с осью лазерного луча и направлена в глубь полубесконечного тела, а радиальная ось $r$ перпендикулярна лучу. Запишем уравнение теплопроводности

$$
\frac{\partial^{2} T}{\partial r^{2}}+\frac{1}{r} \frac{\partial T}{\partial r}+\frac{\partial^{2} T}{\partial z^{2}}=\frac{1}{a} \frac{\partial T}{\partial t}
$$

и дополним его начальными и краевыми условиями:

$$
\left.\frac{\partial T}{\partial z}\right|_{z \rightarrow 0}=-\frac{q}{\lambda} H(R-r),\left.\quad \frac{\partial T}{\partial r}\right|_{r \rightarrow 0}=\left.T\right|_{r \rightarrow \infty}=\left.T\right|_{z \rightarrow \infty}=\left.T\right|_{t \rightarrow 0}=0,
$$

где $\lambda$ - теплопроводность, $a$ - температуропроводность, $H$ - единичная функция Хевисайда. Поверхностная плотность поглощённой мощности выражается через коэффициент поглощения $A$ и полную мощность лазерного луча $P$ : $q=A P /\left(\pi R^{2}\right)$. Первое из условий (2) подразумевает, что величина $q$ внутри пятна нагрева постоянна и равна нулю вне этого пятна.

Виктор Владимирович Манако (к.ф.-м.н., доц.), доцент, каф. общей физики и физики нефтегазового производства. 
В настоящей работе решение краевой задачи $(1),(2)$ представляется в терминах специальных функций, при этом полагается, что величины $\lambda, a, q-$ постоянны.

Дополнительные сведения. Обобщённой гипергеометрической функцией аргумента $x$ называется ряд

$$
{ }_{p} F_{q}\left[\begin{array}{c}
\left(a_{p}\right) \\
\left(b_{q}\right)
\end{array} \mid x\right]=\sum_{k=0}^{\infty} \frac{\left(a_{1}\right)_{k}\left(a_{2}\right)_{k} \ldots\left(a_{p}\right)_{k}}{\left(b_{1}\right)_{k}\left(b_{2}\right)_{k} \ldots\left(b_{q}\right)_{k}} \frac{x^{k}}{k !}=\sum_{k=0}^{\infty} \frac{\prod_{j=1}^{p}\left(a_{j}\right)_{k}}{\prod_{j=1}^{q}\left(b_{j}\right)_{k}} \frac{x^{k}}{k !} .
$$

Здесь $\left(a_{j}\right)_{k}=a_{j}\left(a_{j}+1\right) \ldots\left(a_{j}+k-1\right)=\Gamma\left(a_{j}+k\right) / \Gamma\left(a_{j}\right)$ - символ Похгаммера; $\Gamma(x)$ - гамма-функция Эйлера. Множества чисел $\left(a_{p}\right)$ и $\left(b_{q}\right)$, содержащие соответственно $p$ и $q$ элементов, называются параметрами функции ${ }_{p} F_{q}$.

Большинство стандартных специальных функций - частные случаи функции (3):

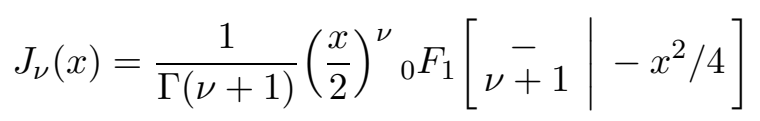

- функция Бесселя первого рода;

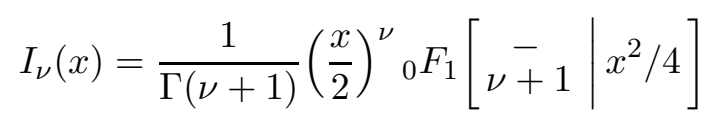

- модифицированная функция Бесселя первого рода;

$$
\Gamma(\nu, x)=\int_{x}^{\infty} \xi^{\nu-1} \exp (-\xi) d \xi=\Gamma(\nu)-\frac{x^{\nu}}{\nu}{ }_{1} F_{1}\left[\begin{array}{c}
\nu \\
\nu+1
\end{array} \mid-x\right]
$$

- неполная гамма-функция;

$$
\begin{aligned}
\mathrm{i}^{n} \operatorname{erfc}(x)=\frac{1}{2^{n} \Gamma(n / 2+1)}{ }_{1} F_{1}\left[\begin{array}{c|c}
-n / 2 & -x^{2} \\
1 / 2 & -
\end{array}\right. & -\frac{2 x}{2^{n} \Gamma((n+1) / 2)}{ }_{1} F_{1}\left[\begin{array}{c}
(1-n) / 2 \\
3 / 2
\end{array}\right.
\end{aligned}
$$

— кратный дополнительный интеграл вероятности $(n \in \mathbb{N})$.

Для гипергеометрической функции аргументов $x, y$ :

$$
F_{l: m ; n}^{p: q ; k}\left[\begin{array}{c}
\left(a_{p}\right):\left(b_{q}\right) ;\left(c_{k}\right) \\
\left(d_{l}\right):\left(f_{m}\right) ;\left(g_{n}\right)
\end{array} \mid x, y\right]=\sum_{r, s=0}^{\infty} \frac{\prod_{j=1}^{p}\left(a_{j}\right)_{r+s} \prod_{j=1}^{q}\left(b_{j}\right)_{r} \prod_{j=1}^{k}\left(c_{j}\right)_{s}}{\prod_{j=1}^{l}\left(d_{j}\right)_{r+s} \prod_{j=1}^{m}\left(f_{j}\right)_{r} \prod_{j=1}^{n}\left(g_{j}\right)_{s}} \frac{x^{r} y^{s}}{r ! s !},
$$

называемой функцией Кампе де Ферье (Kampé de Fériet) [2], можно записать частные случаи:

$$
F_{1: 1 ; 0}^{1: 1 ; 0}\left[\begin{array}{ll}
a: b ;- \\
d: & f ;-
\end{array} \mid x, y\right]=\sum_{k, m=0}^{\infty} \frac{(a)_{k+m}(b)_{k}}{(d)_{k+m}(f)_{k}} \frac{x^{k} y^{m}}{k ! m !}
$$




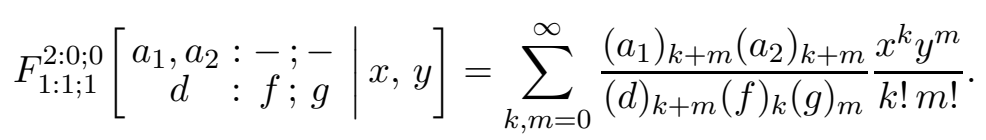

Выпишем функцию Гумберта (Humbert)

$$
\Psi_{2}(a ; f, g ; x, y)=\sum_{k, m=0}^{\infty} \frac{(a)_{k+m}}{(f)_{k}(g)_{m}} \frac{x^{k} y^{m}}{k ! m !}=F_{0: 1 ; 1}^{1: 0 ; 0}\left[\begin{array}{c}
a:-;- \\
-: f ; g
\end{array} \mid x, y\right]
$$

и функцию Аппеля (Appel)

$$
\begin{aligned}
F_{4}\left(a_{1}, a_{2} ; f, g ; x, y\right)=\sum_{k, m=0}^{\infty} \frac{\left(a_{1}\right)_{k+m}\left(a_{2}\right)_{k+m}}{(f)_{k}(g)_{m}} \frac{x^{k} y^{m}}{k ! m !} & = \\
& =F_{0: 1 ; 1}^{2: 0 ; 0}\left[\begin{array}{r}
a_{1}, a_{2}:-;- \\
-\quad: f ; g
\end{array} \mid x, y\right],
\end{aligned}
$$

которые также являются частными случаями функции Кампе де Ферье.

Кроме перечисленных выше функций далее также будет использована общая гипергеометрическая функция Сриваставы (Srivastava), представимая в виде тройного гипергеометрического ряда [3]:

$$
\begin{gathered}
F^{(3)}\left[\begin{array}{c}
(a)::(b) ;\left(b^{\prime}\right) ;\left(b^{\prime \prime}\right):(d) ;\left(d^{\prime}\right) ;\left(d^{\prime \prime}\right) \\
(e)::(g) ;\left(g^{\prime}\right) ;\left(g^{\prime \prime}\right):(h) ;\left(h^{\prime}\right) ;\left(h^{\prime \prime}\right)
\end{array} \mid x, y, z\right]=\sum_{n, k, m=0}^{\infty} \frac{\prod_{j=1}^{A}\left(a_{j}\right)_{n+k+m}}{\prod_{j=1}^{E}\left(e_{j}\right)_{n+k+m}} \times \\
\times \frac{\prod_{j=1}^{B}\left(b_{j}\right)_{n+k} \prod_{j=1}^{B^{\prime}}\left(b_{j}^{\prime}\right)_{k+m} \prod_{j=1}^{B^{\prime \prime}}\left(b_{j}^{\prime \prime}\right)_{n+m} \prod_{j=1}^{D}\left(d_{j}\right)_{n} \prod_{j=1}^{D^{\prime}}\left(d_{j}^{\prime}\right)_{k} \prod_{j=1}^{D^{\prime \prime}}\left(d_{j}^{\prime \prime}\right)_{m}}{\prod_{j=1}^{G}\left(g_{j}\right)_{n+k} \prod_{j=1}^{G^{\prime}}\left(g_{j}^{\prime}\right)_{k+m} \prod_{j=1}^{G^{\prime \prime}}\left(g_{j}^{\prime \prime}\right)_{n+m} \prod_{j=1}^{H}\left(h_{j}\right)_{n} \prod_{j=1}^{H^{\prime}}\left(h_{j}^{\prime}\right)_{k} \prod_{j=1}^{H^{\prime \prime}}\left(h_{j}^{\prime \prime}\right)_{m}} \frac{x^{m} k ! m !}{n !} .
\end{gathered}
$$

Здесь символ $(a)$ обозначает множество чисел, состоящее из $A$ элементов, символ $(b)$ - множество из $B$ элементов и т.д. Частным случаем функции Сриваставы является функция

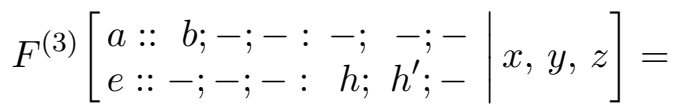

$$
\begin{aligned}
& =\sum_{n, k, m=0}^{\infty} \frac{(a)_{n+k+m}}{(e)_{n+k+m}} \frac{(b)_{n+k}}{(h)_{n}\left(h^{\prime}\right)_{k}} \frac{x^{n} y^{k} z^{m}}{n ! k ! m !} .
\end{aligned}
$$

Расчёт нестационарной температуры. Метод мгновенных точечных источников [4] решение краевой задачи (1), (2) позволяет записать в следующем виде:

$$
\begin{aligned}
T= & 2 q \frac{a}{\lambda} \int_{0}^{t} \frac{d t^{\prime}}{\left(4 \pi a t^{\prime}\right)^{3 / 2}} \int_{0}^{R} r^{\prime} d r^{\prime} \times \\
& \times \int_{0}^{2 \pi} \exp \left(-\frac{\left(r \cos \varphi-r^{\prime} \cos \varphi^{\prime}\right)^{2}+\left(r \sin \varphi-r^{\prime} \sin \varphi^{\prime}\right)^{2}+z^{2}}{4 a t^{\prime}}\right) d \varphi^{\prime} .
\end{aligned}
$$


При $\nu=0$ для функции (4) имеет место [5] интегральное представление

$$
I_{0}(x)=\frac{1}{2 \pi} \int_{0}^{2 \pi} \exp (x \cos \xi) d \xi
$$

позволяющее записать (11) в виде

$$
\begin{aligned}
T=4 \pi q \frac{a}{\lambda} \int_{0}^{t} \frac{d t^{\prime}}{\left(4 \pi a t^{\prime}\right)^{3 / 2}} \times & \\
& \quad \times \int_{0}^{R} \exp \left(-\frac{r^{2}+r^{\prime 2}+z^{2}}{4 a t^{\prime}}\right){ }_{0} F_{1}\left[-\mid\left(\frac{r r^{\prime}}{4 a t^{\prime}}\right)^{2}\right] r^{\prime} d r^{\prime} .
\end{aligned}
$$

Представляя экспоненту и обобщённую гипергеометрическую функцию ${ }_{0} F_{1}$ в виде рядов, можно вычислить следующий определённый интеграл:

$$
\int_{0}^{b} \exp (-a \xi)_{0} F_{1}[\overline{1} \mid \xi] d \xi=b \exp (1 / a) \Psi_{2}(1 ; 1,2 ;-1 / a,-a b) .
$$

При вычислении интеграла учитывались выражения (3), (8), а также первая теорема Куммера [5,6]. Используя полученное равенство, представим (12) в виде

$$
T(r, z, t)=2 \pi r^{2} q \frac{a}{\lambda} \int_{0}^{t} \exp \left(-\frac{z^{2}}{4 a t^{\prime}}\right) \Psi_{2}\left(1 ; 1,2 ;-\frac{r^{2}}{4 a t^{\prime}},-\frac{R^{2}}{4 a t^{\prime}}\right) \frac{d t^{\prime}}{\left(4 \pi a t^{\prime}\right)^{3 / 2}} .
$$

Отсюда, вводя безразмерные величины

$$
\theta=\lambda T /(q R), \quad \tau=4 a t / R^{2}, \quad \sigma=z / R, \quad \rho=r / R,
$$

имеем

$$
\theta(\rho, \sigma, \tau)=\frac{1}{2 \sqrt{\pi}} \int_{1 / \tau}^{\infty} \xi^{-1 / 2} \exp \left(-\sigma^{2} \xi\right) \Psi_{2}\left(1 ; 1,2 ;-\rho^{2} \xi,-\xi\right) d \xi
$$

Раскладывая в ряд функцию (8) и учитывая (3), (5), (9), (10), вычислим интеграл в (13) и получим

$$
\begin{aligned}
\theta(\rho, \sigma, \tau)= & \frac{1}{2 \sigma} F_{4}\left(1 / 2,1 ; 1,2 ;-\rho^{2} / \sigma^{2},-1 / \sigma^{2}\right)- \\
& -\frac{1}{\sqrt{\tau \pi}} F^{(3)}\left[\begin{array}{c}
1 / 2:: 1 ;-;-:-;-;- \\
3 / 2::-;-;-: 1 ; 2 ;-
\end{array}-\rho^{2} / \tau,-1 / \tau,-\sigma^{2} / \tau\right] .
\end{aligned}
$$

Функция (14) является аналитическим решением следующей безразмерной краевой задачи:

$$
\begin{gathered}
\frac{\partial^{2} \theta}{\partial \rho^{2}}+\frac{1}{\rho} \frac{\partial \theta}{\partial \rho}+\frac{\partial^{2} \theta}{\partial \sigma^{2}}=4 \frac{\partial \theta}{\partial \tau} \\
\left.\frac{\partial \theta}{\partial \sigma}\right|_{\sigma \rightarrow 0}=-H(1-\rho),\left.\quad \frac{\partial \theta}{\partial \rho}\right|_{\rho \rightarrow 0}=\left.\theta\right|_{\rho \rightarrow \infty}=\left.\theta\right|_{\sigma \rightarrow \infty}=\left.\theta\right|_{\tau \rightarrow 0}=0 .
\end{gathered}
$$


Заметим, что в работе [4] решение этой краевой задачи, полученное с помощью интегральных преобразований Ханкеля и Фурье, представлено в виде

$$
\begin{aligned}
\theta(\rho, \sigma, \tau)=\frac{1}{2} \int_{0}^{\infty} J_{0}(\rho \xi) J_{1}(\xi)\left[\exp (-\sigma \xi) \operatorname{erfc}\left(\frac{\sigma}{\sqrt{\tau}}-\frac{\xi}{2} \sqrt{\tau}\right)-\right. & \\
& \left.-\exp (\sigma \xi) \operatorname{erfc}\left(\frac{\sigma}{\sqrt{\tau}}+\frac{\xi}{2} \sqrt{\tau}\right)\right] \frac{d \xi}{\xi}
\end{aligned}
$$

Очевидно, что это решение совпадает с (14).

Частные случаи формулы (14). Сделаем ряд замечаний.

$1^{\circ}$. Первое слагаемое в правой части (14) задаёт стационарное температурное поле полубесконечного тела, нагретого круговым источником:

$$
\theta(\rho, \sigma, \infty)=\frac{1}{2 \sigma} F_{4}\left(1 / 2,1 ; 1,2 ;-\rho^{2} / \sigma^{2},-1 / \sigma^{2}\right)
$$

Отметим, что в работе [7] найдено стационарное температурное поле для гауссова источника тепла:

$$
\theta_{\text {гаус }}(\rho, \sigma, \infty)=\Psi_{2}\left(1 / 2 ; 1 / 2,1 ; \sigma^{2},-\rho^{2}\right)-\frac{2 \sigma}{\sqrt{\pi}} \Psi_{2}\left(1 ; 3 / 2,1 ; \sigma^{2},-\rho^{2}\right) .
$$

Расчёт по последним двум формулам даёт ожидаемый результат: модели кругового и гауссова источников тепла предсказывают близкие по значениям температурные поля (см. таблицу).

Заметим, что в соответствии с [8] выражение (15) можно представить в компактной форме:

$$
\theta_{\text {гаус }}(\rho, \sigma, \infty)=\sqrt{2(\rho \pi)^{-1}} V_{0,0}(\rho, \sigma),
$$

\begin{tabular}{c|c|c|c}
\hline$\sigma$ & $\rho$ & $\theta(\rho, \sigma, \infty)$ & $\theta_{\text {гаус }}(\rho, \sigma, \infty)$ \\
\hline 0,01 & 0,01 & 0,990 & 0,989 \\
0,08 & 0,1 & 0,921 & 0,912 \\
0,1 & 0,01 & 0,905 & 0,896 \\
0,08 & 0,6 & 0,827 & 0,783 \\
0,4 & 0,3 & 0,659 & 0,652 \\
0,5 & 0,9 & 0,473 & 0,496 \\
1,0 & 0,0 & 0,414 & 0,428 \\
1,0 & 0,6 & 0,384 & 0,401 \\
1,6 & 0,6 & 0,274 & 0,294 \\
1,6 & 1,2 & 0,242 & 0,265 \\
\hline
\end{tabular}

где $V_{\mu, \nu}(x, y)$ - обобщённая функция Фойгта (Voigt).

$2^{\circ}$. Полагая в формуле (14) $\rho=0$ и учитывая уменьшение количества аргументов для функции Аппеля (см. (3) и (9)):

$$
F_{4}(1 / 2,1 ; 1,2 ; 0, y)={ }_{2} F_{1}\left[\begin{array}{c|c}
1 / 2,1 & y \\
2 & y
\end{array}\right]
$$

и для функции Сриваставы (см. (6) и (10)):

$$
F^{(3)}\left[\begin{array}{c:c:|}
1 / 2:: 1 ;-;-:-;-;- \\
3 / 2::-;-;-: 1 ; 2 ;-
\end{array} \mid 0, y, z\right]=F_{1: 1 ; 0}^{1: 1 ; 0}\left[\begin{array}{c}
1 / 2: 1 ;- \\
3 / 2: 2 ;-
\end{array} \mid y, z\right],
$$

нестационарное температурное поле по оси луча лазера можно записать как $\theta(0, \sigma, \tau)=\frac{1}{2 \sigma}{ }_{2} F_{1}\left[\begin{array}{c|c}1 / 2,1 \\ 2\end{array} \mid-1 / \sigma^{2}\right]-\frac{1}{\sqrt{\tau \pi}} F_{1: 1 ; 0}^{1: 1 ; 0}\left[\begin{array}{c}1 / 2: 1 ;- \\ 3 / 2: 2 ;-\end{array} \mid-1 / \tau,-\sigma^{2} / \tau\right]$. 
Формула (16) совпадает с формулой [4]

$$
\theta(0, \sigma, \tau)=\sqrt{\tau} \operatorname{ierfc}(\sigma / \sqrt{\tau})-\sqrt{\tau} \operatorname{ierfc} \sqrt{\left(\sigma^{2}+1\right) / \tau} .
$$

Однако формула (16) имеет по сравнению с (17) преимущество: в неё входит максимальная (стационарная) температура нагрева на оси лазерного луча в явном виде:

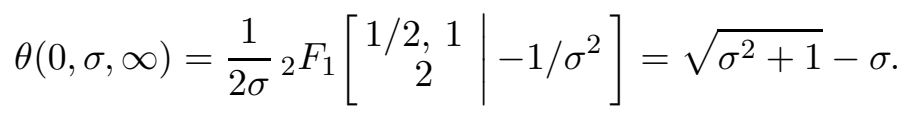

$3^{\circ}$. Рассмотрим нестационарное температурное поле на поверхности полубесконечного тела [4]:

$$
\theta(\rho, 0, \tau)=\int_{0}^{\infty} J_{0}(\rho \xi) J_{1}(\xi) \frac{d \xi}{\xi}-\int_{0}^{\infty} J_{0}(\rho \xi) J_{1}(\xi) \operatorname{erfc}\left(\frac{\xi}{2} \sqrt{\tau}\right) \frac{d \xi}{\xi} .
$$

Стационарную часть (18) можно записать в виде

$$
\begin{aligned}
\theta(\rho, 0, \infty)=\int_{0}^{\infty} J_{0}(\rho \xi) J_{1}(\xi) & \frac{d \xi}{\xi}= \\
& =\left\{\begin{array}{cl}
{ }_{2} F_{1}\left[\begin{array}{c}
-1 / 2,1 / 2 \\
1
\end{array} \mid \rho^{2}\right], & \text { при } \rho \leqslant 1 ; \\
(2 \rho)^{-1}{ }_{2} F_{1}\left[\begin{array}{c}
1 / 2,1 / 2 \\
2
\end{array} \mid \rho^{-2}\right], & \text { при } \rho \geqslant 1 .
\end{array}\right.
\end{aligned}
$$

Значение последнего интеграла, входящего в правую часть (18), можно записать в аналитическом виде, если в формуле (14) для функции Сриваставы $F^{(3)}$ положить $\sigma=0$. При этом функция $F^{(3)}$ за счёт уменьшения количества аргументов вырождается в функцию Кампе де Ферье (7):

$$
\int_{0}^{\infty} J_{0}(\rho \xi) J_{1}(\xi) \operatorname{erfc}\left(\frac{\xi}{2} \sqrt{\tau}\right) \frac{d \xi}{\xi}=\frac{1}{\sqrt{\tau \pi}} F_{1: 1 ; 1}^{2: 0 ; 0}\left[\begin{array}{c}
1 / 2,1:-;- \\
3 / 2: 1 ; 2
\end{array} \mid-\rho^{2} / \tau,-1 / \tau\right] .
$$

Следовательно, формула (18) представима в виде

$$
\theta(\rho, 0, \tau)=\theta(\rho, 0, \infty)-\frac{1}{\sqrt{\tau \pi}} F_{1: 1 ; 1}^{2: 0 ; 0}\left[\begin{array}{c}
1 / 2,1:-;- \\
3 / 2: 1 ; 2
\end{array} \mid-\rho^{2} / \tau,-1 / \tau\right],
$$

где $\theta(\rho, 0, \infty)$ определяется из (19).

$4^{\circ}$. Рассмотрим нестационарное температурное поле на цилиндрической поверхности $\rho=1$, ориентированной соосно лазерному лучу:

$$
\begin{aligned}
\theta(1, \sigma, \tau)=\frac{1}{2 \sigma} F_{4} & \left(1 / 2,1 ; 1,2 ;-1 / \sigma^{2},-1 / \sigma^{2}\right)- \\
& -\frac{1}{\sqrt{\tau \pi}} F^{(3)}\left[\begin{array}{c}
1 / 2:: 1 ;-;-:-;-;- \\
3 / 2::-;-;-: 1 ; 2 ;-
\end{array} \mid-1 / \tau,-1 / \tau,-\sigma^{2} / \tau\right]
\end{aligned}
$$

Данное выражение можно привести к виду 


$$
\begin{aligned}
& \theta(1, \sigma, \tau)=\frac{1}{2 \sigma}{ }_{4} F_{3}\left[\begin{array}{c}
1 / 2,1,1,3 / 2 \\
1,2,2
\end{array} \mid-4 / \sigma^{2}\right]- \\
& -\sqrt{\frac{\tau}{4 \pi}}\left\{F_{1: 1 ; 0}^{1: 1 ; 0}\left[\begin{array}{cc|c}
-1 / 2 & : 1 / 2 ;- \\
1 / 2 & : & 1 ;-
\end{array} \mid-4 / \tau,-\sigma^{2} / \tau\right]-{ }_{1} F_{1}\left[\begin{array}{c}
-1 / 2 \\
1 / 2
\end{array} \mid-\sigma^{2} / \tau\right]\right\},
\end{aligned}
$$

из которого легко выражается стационарная часть:

$$
\begin{gathered}
\theta(1, \sigma, \infty)=\frac{1}{2 \sigma}{ }_{4} F_{3}\left[\begin{array}{c|c}
1 / 2,1,1,3 / 2 & -4 / \sigma^{2} \\
1,2,2 &
\end{array}\right]= \\
\quad=\sqrt{\frac{\sigma^{2}+4}{4}}{ }_{2} F_{1}\left[\begin{array}{c}
-1 / 2,1 / 2 \\
1
\end{array} \mid 4 /\left(\sigma^{2}+4\right)\right]-\frac{\sigma}{2} .
\end{gathered}
$$

Полученный результат совпадает с результатом работы [1], в которой гипергеометрическая функция Гаусса ${ }_{2} F_{1}$ выражается через полный эллиптический интеграл второго рода.

Если в выражении (21) положить $\sigma=0$, то за счёт уменьшения количества аргументов в функции Кампе де Ферье можно записать формулу

$$
\theta(1,0, \tau)=\frac{2}{\pi}-\sqrt{\frac{\tau}{4 \pi}}\left\{{ }_{1} F_{1}\left[\begin{array}{c|c}
-1 / 2 & -4 / \tau \\
1 & -1
\end{array}\right\}\right.
$$

которая позволяет определить температуру на краю поверхностного пятна нагрева. Температура в центре этого пятна вычисляется по формуле

$$
\theta(0,0, \tau)=1-\frac{1}{\sqrt{\tau \pi}}{ }_{2} F_{2}\left[\begin{array}{l}
1 / 2,1 \\
3 / 2,2
\end{array} \mid-1 / \tau\right]
$$

Если здесь применить формулу понижения порядка обобщённой гипергеометрической функции [6]

$$
{ }_{p} F_{q}\left[\begin{array}{c}
\left(a_{p-1}\right), 1 \\
\left(b_{q-1}\right), 2
\end{array} \mid x\right]=x^{-1} \frac{\prod_{j=1}^{q-1}\left(b_{j}-1\right)}{\prod_{k=1}^{p-1}\left(a_{k}-1\right)}\left\{p-1 F_{q-1}\left[\begin{array}{c}
\left(a_{p-1}\right)-1 \\
\left(b_{q-1}\right)-1
\end{array} \mid x\right]-1\right\},
$$

то последнему выражению можно придать вид

$$
\theta(0,0, \tau)=1-\sqrt{\frac{\tau}{\pi}}\left\{{ }_{1} F_{1}\left[\begin{array}{r|r}
-1 / 2 \\
1 / 2
\end{array} \mid-1 / \tau\right]-1\right\}
$$

Формулы (22), (23) могут быть записаны в виде

$$
\begin{gathered}
\theta(1,0, \tau)=\frac{2}{\pi}-\sqrt{\frac{\tau}{4 \pi}}\left\{\exp \left(\frac{-2}{\tau}\right)\left[\left(1+\frac{4}{\tau}\right) I_{0}\left(\frac{2}{\tau}\right)+\frac{4}{\tau} I_{1}\left(\frac{2}{\tau}\right)\right]-1\right\}, \\
\theta(0,0, \tau)=\operatorname{erfc}\left(\frac{1}{\sqrt{\tau}}\right)+\sqrt{\frac{\tau}{\pi}}\left[1-\exp \left(\frac{-1}{\tau}\right)\right]
\end{gathered}
$$

в котором они получены в [1]. 
Заключение. В работе получено аналитическое выражение (14) для нестационарного температурного поля при нагреве полубесконечного тела лучом лазера, создающим на поверхности круговой неподвижный источник тепла. Первое слагаемое в (14) описывает стационарное поле температур и выражается через функцию Аппеля (9), т.е. гипергеометрическую функцию двух аргументов. Второе слагаемое, учитывающее нестационарность процесса, представлено через гипергеометрическую функцию трёх аргументов (функция Сриваставы (10)). Функции (9) и (10) допускают уменьшение количества аргументов в трёх случаях: на оси лазерного луча (см. формулу (16)), на свободной поверхности (см. формулу (20)) и на круговой цилиндрической поверхности, являющейся продолжением границы зоны нагрева (см. формулу (21)).

Рассмотрены стационарные температурные поля, образуемые круговым и гауссовым источниками. Существенных количественных различий в этих полях не обнаружено.

Автор выражает искреннюю благодарность В. А. Пинскеру, сделавшему ценные и полезные замечания после прочтения рукописи данной работы.

\section{БИБЛИОГРАФИЧЕСКИЙ СПИСОК}

1. Пинскер В. А. Нестационарное температурное поле в полуограниченном теле, нагреваемом круговым поверхностным источником тепла // Теплофизика высоких температур, 2006. T. 44, № 1. C. 127-135; англ. пер.: Pinsker V. A. Unsteady-state temperature field in a semi-infinite body heated by a disk surface heat source // High Temperature, 2006. Vol. 44, no. 1. Pp. 129-138.

2. Appel P., Kampé de Fériet J. Fonctions Hypergéométriques et Hypersphériques. Polynômes d'Hermite. Paris: Gauthier-Villars, 1926. 202 pp.

3. Srivastava H.M., Manocha H.L. A treatise on generating functions. New York: Halsted Press [John Wiley \& Sons, Inc.], 1984. 569 pp.

4. Carslaw H.S., Jaeger J. C. Conduction of Heat in Solids. Oxford: Clarendon Press, 1959. 510 рр.; русск. пер.: Карслоу Г., Егер Д. Теплопроводность твёрдых тел. М.: Наука, 1964. $488 \mathrm{c}$.

5. Handbook of Mathematical Functions with Formulas, Graphs, and Mathematical Tables / eds. M. Abramowitz, I. A. Stegun. New York: Dover, 1972. 824 pp.; русск. пер.: Справочник по специальным функциям / ред. М. Абрамовиц, И. Стиган. М.: Наука, 1979. $832 \mathrm{c}$.

6. Прудников А.П., Брычков Ю.А., Маричев О.И. Интегралы и ряды. Т. 3: Специальные функции. Дополнительные главы. М.: Физматлит, 2003. 688 с. [Prudnikov A.P. Brychkov Yu.A., Marichev O.I. Integrals and series. Vol.3: Special functions. Supplementary chapters. Moscow: Fizmatlit, 2003. 688 pp.]

7. Манако В.В., Путилин В.А., Камашев А. В. Расчет температуры при нагреве неподвижным лазерным лучом// Теплофизика высоких температур, 2011. Т. 49, № 1. C. 126-132; англ. пер.: Manako V.V., Putilin V.A., Kamashev A. V. Calculation of temperature under heating by an immobile laser beam // High Temperature, 2011. Vol. 49, no. 1. Pp. 127-134.

8. Srivastava H. M., Miller E. A. A unified presentation of the Voigt functions // Astrophys. and Space Sci., 1987. Vol. 135, no. 1. Pp. 111-118.

Поступила в редакцию 11/XI/2011; в окончательном варианте - 16/IV/2012. 
MSC: 35Q80; 80A20, 33C70

A REPRESENTATION IN TERMS OF HYPERGEOMETRIC FUNCTIONS FOR THE TEMPERATURE FIELD IN A SEMI-INFINITE BODY THAT IS HEATED BY A MOTIONLESS LASER BEAM

\section{V. Manako}

Samara State Technical University,

244, Molodogvardeyskaya st., Samara, 443100, Russia.

E-mail: viktor.manako@mail.ru

We have considered an analytical expression for the temperature field of a semi-infinite body that is heated by a circular heat source located at the free surface. Unsteady temperature field is expressed in terms of the Appell and the Srivastava hypergeometric functions. We have studied some special areas in heated body where a non-stationary temperature field is expressed in terms of the Kampé de Fériet function. The obtained expressions have allowed to carry out the separation of the stationary and non-stationary parts of temperature field from each other. Calculations of the steady temperature fields generated by circular or Gaussian sources have been accomplished. Significant quantitative differences in these fields were not found.

Key words: heat conductivity boundary value problem, circular heat source, hypergeometric series.

Original article submitted 11/XI/2011; revision submitted $16 / \mathrm{IV} / 2012$.

Victor V. Manako (Ph. D. (Phys. \& Math.)), Associate Professor, Dept. of General Physics and Physics of Oil and Gas Production. 